\title{
Stigma towards People Living on HIV/AIDS and Associated Factors among Nurses' Working in Amhara Region Referral Hospitals, Northwest Ethiopia: A Cross-Sectional Study
}

\author{
Mohammed Hassen Salih, ${ }^{1}$ Gizachew Assefa Tessema, ${ }^{2}$ Endeshaw Admassu Cherkos, \\ Abebaw Jember Ferede, ${ }^{1}$ and Degefaye Zelalem Anlay ${ }^{1}$ \\ ${ }^{1}$ School of Nursing, College of Medicine \& Health Sciences, University of Gondar, Gondar, Ethiopia \\ ${ }^{2}$ Department of Reproductive Health, Institute of Public Health, College of Medicine \& Health Sciences, University of Gondar, \\ Gondar, Ethiopia \\ ${ }^{3}$ Department of Midwifery, College of Medicine \& Health Sciences, University of Gondar, Gondar, Ethiopia
}

Correspondence should be addressed to Mohammed Hassen Salih; muhenet@gmail.com

Received 15 January 2017; Revised 23 February 2017; Accepted 7 March 2017; Published 19 March 2017

Academic Editor: Jenny M. Wilkinson

Copyright @ 2017 Mohammed Hassen Salih et al. This is an open access article distributed under the Creative Commons Attribution License, which permits unrestricted use, distribution, and reproduction in any medium, provided the original work is properly cited.

\begin{abstract}
Introduction. HIV/AIDS-related stigma occurs in the world towards people living with HIV/AIDS in a different form. Stigma among nurses in health care setting is one of the main challenges towards the prevention and management of HIV/AIDS in developing countries. It is one of the main reasons keeping patients from seeking health care service. Therefore assessing the magnitude of stigma and associated factors towards people living on HIV/AIDS among nurses is of paramount importance for the quality of nursing care as well as service utilization. Methods. An institutional based cross-sectional study was conducted in March 2013. Pretested and structured questionnaire via self-administration was used in the tool of HIV/AIDS Stigma Instrument-Nurse (HASIN). Data were entered using EPI info version 3.5.3 and transferred to SPSS version 20 for further analysis. Descriptive statistics were conducted to summarize the sample characteristics. A backward stepwise logistic regression model was fitted and adjusted odds ratio with 95\% confidence interval was calculated to identify associated factors. Results. A total of 386 nurses participated yielding a response rate of $97.2 \%$. Nearly two-thirds (64.5\%) of them have shown stigma towards people living with HIV/AIDS in the health institution. Qualification level of diploma or certificate, lack of training, experiences of $<06$ years, low HIV patient caseload seen in the last six months, and the absence of guidelines/protocols about HIV/AIDS in their health institution were associated factors for stigma. Conclusions. The findings of this research showed high magnitude of stigma towards people living with HIV/AIDS among nurses. For stigma to be decreased nurses need to update their knowledge through training and experience sharing with senior staff. And it is crucial that the Ethiopian Ministry of Health, Amhara Regional Health Bureau, and the two hospitals work for decreasing stigma by creating educational development, ensuring accessibility of guidelines about HIV/AIDS, and providing access to training.
\end{abstract}

\section{Background}

The United Nations Program on HIV/acquired immune deficiency syndrome (UNAIDS) reported that globally there were an estimated 35.3 (32.2-38.8) million people who were living with HIV in 2013. Even if the proportion of countries documenting the existence of HIV-related legal services increased from $45 \%$ in 2008 to $55 \%$ in 2012 stigma occurs by different means. It is still one of the major challenges for management and control of HIV/AIDS epidemic [1, 2]. The incidence of HIV in Ethiopia reduced by $90 \%$ between 2001 and 2012. With this significant reduction the current incidence and prevalence of HIV are still a concern for the government as well as community $[3,4]$. According to an Ethiopian Demographic and Health Survey (EDHS) report, the HIV prevalence among adults aged groups (15-49 ys) was $1.5 \%$; specifically the prevalence in Amhara region is almost in line with a national prevalence $1.6 \%$ [5]. 
In addition to the medical problems people living on HIV/AIDS (PLHA) face different problems including social problems, like stigma. "A stigma has been defined as a miss identity whereby a person or a group is seen as being inferior because of an attribute or disease they have such as HIV/ AIDS." Even though the presentation worldwide is different across the countries and organizations, HIV/AIDS-related stigma by health professionals is still high [6, 7]. It also remains one of the barriers for effective prevention and management of HIV/AIDS.

Stigma on HIV patient is shown to affect the quality of life of the patient especially when it is experienced by health professionals like nurse and other health care workers, since their access to care and adherence to the medication might be affected.

However, the extent of stigmatization towards PLHA by nurses' is not fully understood. Different researchers have tried to find the main reason and factors of why nurses' demonstrate stigma particularly towards PLHA. The major reasons identified by different research are lack of in-depth knowledge [8-10], the perception of incurable natures of HIV [8], fear of being infected with HIV $[10,11]$, the notion that HIV is transmitted through sexual intercourse [10], and lack of orientation regarding polices against $\operatorname{HIV}[8,9,11]$.

In Africa, even if the most important activities of health care services are delivered by nurses, there is limited published data that explores the relationship between stigma towards PLHA and nursing professionals $[12,13]$. In Ethiopia, at different time, researchers have tried to address the impact of stigma towards PLHA in health institutions by health professionals, including nurses. However, it was difficult to find full data that shows the magnitude of stigma, especially by nurses [14].

This research will be a point of departure for further study. Moreover, it will help planners and policy makers to design best interventions towards the control and prevention of stigma towards PLHA.

\section{Materials and Methods}

An institution based cross-sectional study design was conducted among nurses working at Amhara Region Referral Hospitals in Northwest Ethiopia. In Amhara region northwest direction there are two referral hospitals, namely, Gondar University Hospital and Felege Hiwot Referral Hospital.

Gondar University Hospital is one of the tertiary level hospitals located in Gondar town, Northwest Ethiopia, $740 \mathrm{~km}$ away from Addis Ababa. There are 520 beds and about 448 Health Care Workers (HCWs) of whom 255 were nurses. Nurses are assigned to all departments of the hospital. The Felege Hiwot referral Hospital is also a tertiary level and referral hospital located in Bahir Dar town, Northwest Ethiopia, $554 \mathrm{Km}$ away from Addis Ababa. The hospital has 300 beds and approximately 142 nurses. These hospitals are rendering service for more than 5 million of the population in their respective catchment area. The study was conducted at March 2013 GC.
All nurses who are working in these hospitals were the source population and those nurses who were avail during the study period were included in this study.

The sample size was calculated based on a single proportion formula by using the assumption of $95 \%$ confidence interval, $50 \%$ of single population proportion (because there is no study in the area), and considering 5\% margin of error to yield the final sample size of 384 . However, the numbers of nurses who are working in both hospitals were 397. Therefore all nurses were included.

The dependent variable that is stigma was measured by a validated tool (HIV/AIDS Stigma Instrument-Nurse (HASIN)) which was prepared by the study conducted in three phases in five African countries (Lesotho, Malawi, South Africa, Swaziland, and Tanzania) from 2004 to 2006. It is defined as those nurses having scored one (once) and above in the tool in the last three months [9].

Perceived institutional support is one of the independent variables/factors we used in this research; it is defined as the perception of nurses regarding their institutional support in relation to supply or equipment, protocols, policy to prevent stigma in health institution, and implementation of that policy. Also the number of patients who received services by nurses for the past six months was grouped into two; delivered services for less than ten HIV/AIDS patients in the last six months were grouped into low HIV/AIDS caseload and delivered services for 10 or more patients in the last six months were grouped into high HIV/AIDS caseload. This grouping was supported by other research conducted in Southern Ethiopia [15].

Data were collected by using structured, pretested and self-administered English version questionnaire. Four B.S. nurses have facilitated the data collection process.

For dependent variable, the HASI-N questionnaire, the participants were asked to note how often they have notified the stigma during the past 3 months ( 0 : never, 1 : once or twice, 2: several times, or 3: most of the time). The responses for each item were grouped into two: never ( 0 ) for no nurses notifying the stigma, and from once to most of the time was grouped into a yes (1) for nurses notifying the stigma.

Data were entered by EPI info version 3.5.3 and exported to SPSS version 20 statistical package for further analysis. Using binary logistic regression for each independent variable against dependent variable, the strength of the association and their statistical significance (OR) and 95\% confidence interval was calculated. Those variables having $P$ values less than 0.2 in bivariate analysis were entered into the multivariate analysis using backward likelihood ratio method. The final result was interpreted based upon their association of significance (i.e., $P$ value is below or equal to 0.05 ).

Ethical clearance was obtained from an institutional review committee of College of Medicine and Health Sciences, University of Gondar. Communication with Gondar University Referral Hospital and Felege Hiwot Referral Hospital administrator was made through an official letter from the School of Nursing. The purposes and the importance of the study were explained to each study participant and then written informed consent was secured. Confidentiality 
was maintained at all levels of the study by making the data collection anonymous.

\section{Results}

3.1. Sociodemographic and Personal Characteristics of Study Participants. A total of 386 of nurses with a response rate of $97.2 \%$ returned the questionnaire. About half $(50.5 \%)$ of them were females and married. The mean age of respondents was 28.8 years $(\mathrm{SD}=06$ years). Nearly half of them $(44.8 \%)$ were in the age group of 25-29 years. The mean monthly income was 2213 ETB (SD = 676.7); more than half, 197 (51\%), had a monthly income of 2250 ETB or more. The majority $(82.9 \%)$ of nurses was orthodox in their religion (Table 1).

Concerning perceived religion most of the nurses 229 $(59.3 \%)$ were perceived somewhat religious. Nearly twothirds of nurses $(61.9 \%)$ had a first degree. The median year of experience of nurses was 05 years $(\mathrm{IQR}=06)$. More than half (54.1\%) of them had an experience of less than or equal to five years. Two hundred and sixteen (56\%) of respondents did not attend training regarding the prevention of stigma towards HIV/AIDS. Regarding HIV/AIDS-related health care service $263(68.1 \%)$ of nurses delivered care in the past six months. Most of them provided care to a group of 10 or more patients (median $=13$, IQR $=14)$ (Table 2$)$.

Two-thirds $(66.3 \%)$ of the nurses mentioned that they had an equipment/supply in their institution which helps to prevent stigma in their institution, followed by implementation of policy related to institutional support, and guideline/protocols-related institutional support.

From the total of 386 nurses who were participating in the study, about two-thirds (64.5\%) of nurses perceived stigma towards PLHA in the last three months in their health institutions. The stigma scores ranged from once to most of the time, with total mean and standard deviation of 0.65 and \pm 0.48 , respectively (Table 2 ).

3.2. Factors Associated with Stigma towards PLHA. In the bivariate logistic regression analysis, stigma was significantly associated with age, sex, monthly income, qualification, experience, training related prevention of stigma, giving health care service, and numbers of HIV/AIDS patients caseload for the last six months. However, in the multivariate logistic regression analysis qualification level, experience, the presence of training including prevention of stigma of HIV/AIDS and HIV patient caseload was associated with the stigma (Table 3).

Those nurses who had diploma or certificates were about 3.6 times $[\mathrm{AOR}=3.63,95 \% \mathrm{CI}(1.27,10.33)]$ more likely to have a stigma towards PLHA as compared to those having the first degree or above. Nurses who had 5 years or lesser year of experience were 5.32 times $[\mathrm{AOR}=5.32,95 \% \mathrm{CI}(2.01,14.08)]$ more likely to have stigma than those who experienced 5 years or more. Those who did not receive any training on stigma prevention towards PLHA were about 4 times $[\mathrm{AOR}=$ $3.94,95 \%$ CI $(1.51,10.27)]$ more likely to have a stigma as compared to those who had training. Moreover, those nurses who were perceived as low HIV caseload for delivering service in the last six months by nurses were 4.6 times $[\mathrm{AOR}=4.64$,
TABLE 1: Sociodemographic characteristics of nurses, working at Amhara Region Referral Hospitals, Northwest Ethiopia, 2013 ( $n=$ 386).

\begin{tabular}{lccc}
\hline Variables & Frequency & Percentage & Mean (SD) \\
\hline Age in years & & & $28.8(6)$ \\
$20-24$ & 85 & 22 & \\
$25-29$ & 173 & 44.8 & \\
$30-34$ & 74 & 19.2 & \\
$>34$ & 54 & 14 & \\
Sex & & & \\
Male & 191 & 49.5 & \\
Female & 195 & 50.5 & \\
Marital status & & & \\
Single & 178 & 46.1 & \\
Married & 199 & 51.6 & \\
Divorced/separated & 9 & 2.3 & \\
Monthly salary (ETB) & & 12.2 & \\
1416 ETB or below & 47 & 36 & \\
1417-2249 ETB & 139 & 51.8 \\
2250 or above & 200 & 82.9 \\
Religion & & 12.2 \\
Orthodox & 320 & 4.7 \\
Muslim & 47 & 0.3 \\
Protestant & 18 & \\
Catholic & 1 & \\
\hline
\end{tabular}

ETB: Ethiopian Birr, SD: standard deviation.

95\% CI $(1.57,13.70)]$ more likely to have stigma than those who give nursing care or other health services grouped as a high case load in the last six months.

Concerning perceived institutional support all four variables, which were equipment, policy on the protection of those diagnosed with HIV/AIDS from stigma in health institutions, the implementation of the policy, and presence of guidelines or protocols for prevention of stigma, they were significantly associated with stigma increment in the bivariate logistic regression analysis. However, only perceived guidelines or protocols were significantly associated with stigma in multivariate analysis. Those who were unaware of the presence of guidelines or protocols were 4 times [AOR = $4.19,95 \%$ CI $(1.31,13.41)]$ more likely to have a stigma towards PLHA as compared to those who were aware of protocols or guidelines (Table 3 ).

\section{Discussion}

Stigma observed among nurses towards PLHA during delivering health service in health institution is one of the main challenges in the prevention and management of HIV/AIDS in developing countries. This study was aimed to assess the magnitude and associated factors of stigma towards PLHA among nurses working at Amhara region Referral Hospitals in Northwest area.

The magnitude of stigma among nurses towards PLHA was $64.5 \%$. This result is lower than the study conducted 
TABLE 2: Personal characteristics and stigma score of nurses, working at Amhara Region Referral Hospitals, Northwest Ethiopia, 2013 ( $n=$ 386).

\begin{tabular}{|c|c|c|c|}
\hline Variable & Frequency & Percentage & Median (IQR) \\
\hline \multicolumn{4}{|l|}{ Perceived religiosity } \\
\hline Very religious & 137 & 35.5 & \\
\hline Somewhat religious & 229 & 59.3 & \\
\hline Not religious & 20 & 5.2 & \\
\hline \multicolumn{4}{|l|}{ Professional qualification } \\
\hline Certificate/diploma & 135 & 35 & \\
\hline First degree & 251 & 65 & \\
\hline Years of experience & & & $5(6)$ \\
\hline 7 months up to 5 years & 209 & 54.1 & \\
\hline Greater than 5 years & 177 & 45.9 & \\
\hline \multicolumn{4}{|l|}{ Training about stigma } \\
\hline Yes & 170 & 44 & \\
\hline No & 216 & 56 & \\
\hline \multicolumn{4}{|l|}{ Giving health care service for PLHA in the last six months } \\
\hline Yes & 263 & 68.1 & \\
\hline No & 123 & 31.9 & \\
\hline Numbers of HIV/AIDS clients seen in the last six months $(n=263)$ & & & $13(14)$ \\
\hline Low caseloads (less than 10 HIV/AIDS clients) & 94 & 35.7 & \\
\hline High caseloads (10 or more than 10 HIV/AIDS clients) & 169 & 64.3 & \\
\hline Stigma & & & $0.65(0.48)$ \\
\hline Yes & 249 & 64.5 & \\
\hline No & 137 & 35.5 & \\
\hline
\end{tabular}

IQR: Interquartile Range, PLHA: People living with HIV/AIDS.

in five African countries, $80.3 \%$ [16], albeit higher than the studies conducted in India (55\%) and the USA, ranged from $29 \%$ to $41 \%$ on those HASI-N tool components [17, 18]. The possible reasons for the difference may be due to the difference in health institution structure such as infection prevention procedures, organizational systems to decrease the risk of exposure from HIV/AIDS, resource utilization, or availability in health sectors and differences in professional safety. Also, in the studies conducted in India the inclusion of health professionals was extended to other health professionals such as physicians and laboratory technicians.

Those professionals having certificate/diploma were about 3.6 times more likely to have a stigma as compared to those having a degree or above. The finding is consistent with studies conducted in Southern Ethiopia and China [9, 19]. The possible reasons might be due to the difference in job description which in turn leads to different working environment that might create stigma and less access to further study; those nurses might be assigned in more challenged and have less access to HIV/AIDS care strategies, in addition to decreased awareness and knowledge about modes of transmission and risk of exposure types seen in their lower educational curriculum.

Those nurses with 05 years' experience or less were more than five times more likely to have a stigma as compared to those who had above 05 years' experience in their profession. This result is consistent with studies conducted in China and
Ethiopia [9, 19]. This could be due to the fact that more experienced nurses had more exposure to, and awareness of, HIV/AIDS management and prevention. However, the finding contradicts the findings of medical professionals of Southeast China and Turkish nurses and midwives [11, 20]. The possible reason for the difference may be due to the difference in their professional qualification or perhaps some nurses had a little patient contact in their assigned unit/ward.

Those who were not trained on awareness of stigma towards HIV/AIDS were about four times more likely to experience stigma than those who had training. This finding is in line with the studies conducted in Southern Ethiopia, Cuba, and China $[9,19,21]$. The possible reason may be due to increased knowledge gained by training having an impact on control of stigma towards PLHA. Receiving training on stigma might not directly impact on nurses' stigmatizing behavior at work, but it can be an influence on nurses' general stigma towards PLHA.

Those who were perceived to have low HIV caseload were about 4.6 times more likely to show HIV/AIDS-related stigma as compared to those who were perceived as having a high caseload in the last six months. This result is consistent with a study conducted in Southern Ethiopia and Barbados $[9,22]$. The possible explanations could be that, with increased exposure to a number of PLHA on an everyday basis, this may lead to close contact with the patient and result in more experience and increased confidence in how to 
TABLE 3: Bivariate and multivariate analysis of factors associated with stigma towards PLHA among nurses, working in Amhara Region Referral Hospitals, Northwest Ethiopia, $2013(n=386)$.

\begin{tabular}{|c|c|c|c|c|}
\hline \multirow{2}{*}{ Variables } & \multicolumn{2}{|c|}{ Stigma } & \multirow{2}{*}{$\begin{array}{c}\text { Crude OR } \\
(95 \% \text { CI })\end{array}$} & \multirow{2}{*}{$\begin{array}{c}\text { Adjusted OR } \\
(95 \% \mathrm{CI})\end{array}$} \\
\hline & Yes & No & & \\
\hline \multicolumn{5}{|l|}{ Age in years } \\
\hline $20-24$ & 69 & 16 & $8.63(3.94,18.91)$ & \\
\hline $25-29$ & 136 & 37 & $7.35(3.75,14.40)$ & \\
\hline $30-34$ & 26 & 48 & $1.08(0.52,2.27)$ & \\
\hline 35 or more & 18 & 36 & 1 & \\
\hline \multicolumn{5}{|l|}{ Sex } \\
\hline Male & 116 & 75 & $0.72(0.47,1.10)$ & \\
\hline Female & 133 & 62 & 1 & \\
\hline \multicolumn{5}{|l|}{ Monthly salary (ETB) } \\
\hline 1416 or below & 39 & 8 & $3.53(1.57,7.94)$ & \\
\hline $1417-2249$ & 94 & 45 & $1.51(0.96,2.38)$ & \\
\hline 2250 or more & 116 & 84 & 1 & \\
\hline \multicolumn{5}{|l|}{ Qualification } \\
\hline Diploma/certificated & 112 & 23 & $4.05(2.43,6.77)$ & $3.63(1.27,10.33)$ \\
\hline Degree and above & 137 & 114 & 1 & 1 \\
\hline \multicolumn{5}{|l|}{ Experience } \\
\hline 07 months -05 Yrs & 174 & 35 & $6.76(4.23,10.82)$ & $5.32(2.01,14.08)$ \\
\hline Greater than 05 Yrs & 75 & 102 & 1 & 1 \\
\hline \multicolumn{5}{|l|}{ Training } \\
\hline Getting training & 78 & 92 & 1 & 1 \\
\hline Not getting training & 171 & 45 & $4.48(2.87,7.00)$ & $3.94(1.51,10.27)$ \\
\hline \multicolumn{5}{|l|}{ Giving health care service } \\
\hline Yes & 161 & 102 & 1 & \\
\hline No & 88 & 35 & $1.59(1.00,2.53)$ & \\
\hline \multicolumn{5}{|l|}{ HIV case load } \\
\hline Low HIV caseload & 79 & 15 & $5.59(2.98,10.48)$ & $4.64(1.57,13.70)$ \\
\hline High HIV caseload & 82 & 87 & 1 & 1 \\
\hline \multicolumn{5}{|l|}{ Supply/equipment IS } \\
\hline Yes & 150 & 106 & 1 & \\
\hline No & 99 & 31 & $2.26(1.41,3.63)$ & \\
\hline \multicolumn{5}{|c|}{ Policy to protect HIV/AIDS patients } \\
\hline Yes & 119 & 79 & 1 & \\
\hline No & 130 & 58 & $1.49(0.98,2.27)$ & \\
\hline \multicolumn{5}{|l|}{ Implementation of policy } \\
\hline Yes & 70 & 56 & 1 & \\
\hline No & 49 & 23 & $1.70(0.93,3.13)$ & \\
\hline \multicolumn{5}{|l|}{ Guidelines/protocols IS } \\
\hline Yes & 125 & 110 & 1 & 1 \\
\hline No & 124 & 27 & $4.04(2.48,6.59)$ & $4.19(1.31,13.41)$ \\
\hline
\end{tabular}

OR: odd ratio, ETB: Ethiopian Birr, and IS: institutional support.

manage this client group. Moreover, those nurses with high caseloads are most likely to have been assigned to the antiretro viral therapy (ART) service unit and will have better knowledge of HIV/AIDS than others.
Those who were not aware of the presence of protocols were four times more likely to show stigma than those who were unaware of the presence of protocols or guidelines. This result is in line with a study conducted in Cuba [21] and 
Southern Ethiopia [9]. The possible reasons may be a lack of specific protocols or guidelines leading to nurses perceiving more stigmatization than others. Also, those who were aware of the presence of protocols or guidelines may have received more training and were, therefore, aware of stigma reduction strategies such as the use of protocols and guideline manuals.

The study has several limitations. First, the cross-sectional design of this study limits our ability to make causal inferences. Second, since this study is done on nurse professionals only it is difficult to compare this finding across different professionals.

\section{Conclusion}

In general, this study shows that the magnitude of stigma towards PLHA among nurses is found to be high. Professional level diploma/certificates, an experience of less than or equal to 5 years, not receiving training, low HIV caseloads in the last six months, and not being aware of institutional support in the form of guidelines were associated factors for stigma towards PLHA. Even if all nurses included in this research are working in the resource limited country and have difficulties in their working environment, nurses needs to pay attention to providing quality of nurses care for PLHA.

Due to stigma, the quality of nursing care towards PLHA may not fully address their health care needs. Giving supportive monitoring, close mentoring to all levels of nurses, ensuring the availability of guidelines and protocols, and creating opportunities for nurses to attend different seminars and HIV/AIDS training are recommended to decrease stigmatization among nurses.

\section{Conflicts of Interest}

The authors declare that they have no competing interests.

\section{Authors' Contributions}

Mohammed Hassen Salih wrote the proposal, participated in data collection, analyzed the data, and drafted the paper. Gizachew Assefa Tessema and Endeshaw Admassu Cherkos approved the proposal with revision; Abebaw Jember Ferede and Degefaye Zelalem Anlay participated in data analysis and revised subsequent drafts of the paper. All authors read and approved the final manuscript.

\section{Acknowledgments}

The authors would like to express their deepest gratitude to the University of Gondar for the approval of the ethical clearance and for their technical and financial support of this study. They would also like to thank all nurses who participated in this study for their commitment in responding to their questionnaire.

\section{References}

[1] WHO, WHO Global Fact Sheet 2011, WHO, Geneva, Switzerland, 2012.
[2] UNAIDS Corporate publications. 20 Avenue Appia $\mathrm{CH}-$ 1211, Geneva 27, Switzerland: World Health organization, 2013, http://apps.who.int/iris/bitstream/10665/85326/1/9789241505734_ eng.pdf.

[3] UNAIDS, World AIDS Report, 2012, http://www.unaids.org/ sites/default/files/media_asset/JC2434_WorldAIDSday_results_ en_1.pdf.

[4] WHO, “Global fact sheet 2011," December 2012, http://www .unaids.org/en/resources/publications/2012/.

[5] Ethiopia Demographic and Health Survey 2011, Central Statistical Agency and ICF International, Addis Ababa, Ethiopia, 2011.

[6] N. C. Mbonu, B. van den Borne, and N. K. De Vries, "Stigma of people with HIV/AIDS in Sub-Saharan Africa: a literature review," Journal of Tropical Medicine, vol. 2009, Article ID 145891, 14 pages, 2009.

[7] N. Rosenburg, D. Taliaferro, and P. Ercole, "HIV-related stigma among nursing students in cameroon," Journal of the Association of Nurses in AIDS Care, vol. 23, no. 2, pp. 170-176, 2012.

[8] C. Lin, L. Li, D. Wan, Z. Wu, and Z. Yan, "Empathy and avoidance in treating patients living with HIV/AIDS (PLWHA) among service providers in China," AIDS Care, vol. 24, no. 11, pp. 1341-1348, 2012.

[9] G. T. Feyissa, L. Abebe, E. Girma, and M. Woldie, "Stigma and discrimination against people living with HIV by healthcare providers, Southwest Ethiopia," BMC Public Health, vol. 12, no. 1, article 522, 2012.

[10] A. K. M. Ahsan Ullah, "HIV/AIDS-related stigma and discrimination: a study of health care providers in Bangladesh," Journal of the International Association of Physicians in AIDS Care, vol. 10, no. 2, pp. 97-104, 2011.

[11] M. Akgun Kostak, S. Unsar, S. Kurt, and O. Erol, "Attitudes of Turkish midwives and nurses working at hospitals towards people living with human immunodeficiency virus/acquired immune deficiency syndrome," International Journal of Nursing Practice, vol. 18, no. 5, pp. 437-444, 2012.

[12] L. R. Uys, W. L. Holzemer, M. L. Chirwa et al., "The development and validation of the HIV/AIDS Stigma Instrument-Nurse (HASI-N)," AIDS Care, vol. 21, no. 2, pp. 150-159, 2009.

[13] W. L. Holzemer, L. R. Uys, M. L. Chirwa et al., "Validation of the HIV/AIDS stigma instrument-PLWA (HASI-P)," AIDS Care, vol. 19, no. 8, pp. 1002-1012, 2007.

[14] Y. Assefa, A. Kiflie, B. Tekle, D. H. Mariam, M. Laga, and W. Van Damme, "Effectiveness and acceptability of delivery of antiretroviral treatment in health centres by health officers and nurses in Ethiopia," Journal of Health Services Research and Policy, vol. 17, no. 1, pp. 24-29, 2012.

[15] G. T. Feyissa, L. Abebe, E. Girma, and M. Woldie, "Validation of an HIV-related stigma scale among health care providers in a resource-poor Ethiopian setting," Journal of Multidisciplinary Healthcare, vol. 5, pp. 97-113, 2012.

[16] W. L. Holzemer, L. N. Makoae, M. Greeff et al., "Measuring HIV stigma for PLHAs and nurses over time in five African countries," SAHARA-J: Journal of Social Aspects of HIV/AIDS, vol. 6, no. 2, pp. 76-82, 2009.

[17] V. S. Mahendra, L. Gilborn, S. Bharat et al., "Understanding and measuring AIDS-related stigma in health care settings: a developing country perspective," SAHARA-J: Journal of Social Aspects of HIV/AIDS, vol. 4, no. 2, pp. 616-625, 2007.

[18] I. P. Williams and L. Searcy, "Study: is bedside nursing still affected by HIV stigma? A peer-review article," HIV Clinician, vol. 24, no. 4, pp. 9-10, 2012. 
[19] L. Li, Z. Wu, S. Wu, Y. Zhaoc, M. Jia, and Z. Yan, "HIV-related stigma in health care settings: a survey of service providers in China," AIDS Patient Care and STDs, vol. 21, no. 10, pp. 753762, 2007.

[20] G. Cai, K. Moji, S. Honda, X. Wu, and K. Zhang, "Inequality and unwillingness to care for people living with HIV/AIDS: a survey of medical professionals in southeast China," AIDS Patient Care and STDs, vol. 21, no. 8, pp. 593-601, 2007.

[21] A. Andrewin and L.-Y. Chien, "Stigmatization of patients with HIV/AIDS among doctors and nurses in Belize," AIDS Patient Care and STDs, vol. 22, no. 11, pp. 897-906, 2008.

[22] E. Massiah, T. C. Roach, C. Jacobs et al., "Stigma, discrimination, and HIV/AIDS knowledge among physicians in Barbados," Revista Panamericana de Salud Pública, vol. 16, no. 6, pp. 395-401, 2004. 


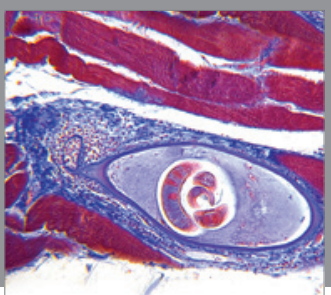

Gastroenterology

Research and Practice
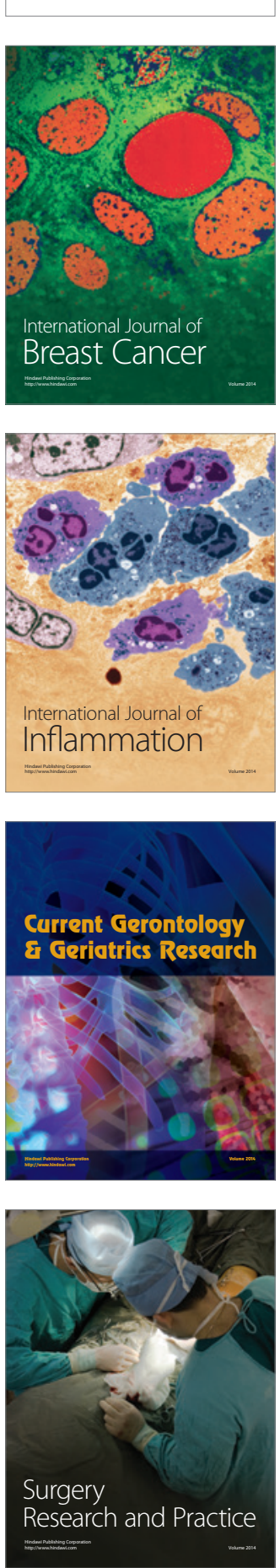

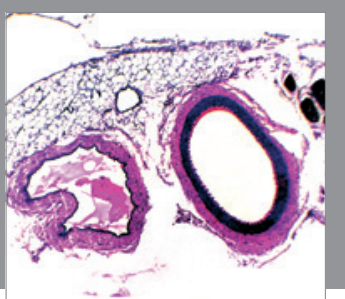

International Journal of Hypertension
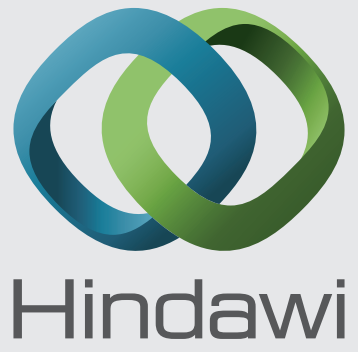

Submit your manuscripts at

https://www.hindawi.com
The Scientific Nursing

World Journal Research and Practice

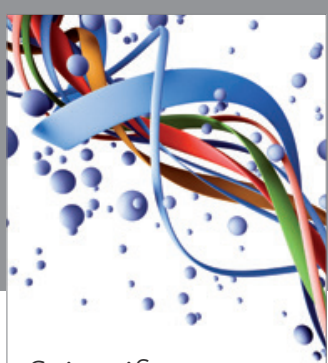

Scientifica

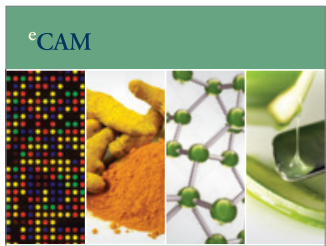

Evidence-Based

Complementary and

Alternative Medicine
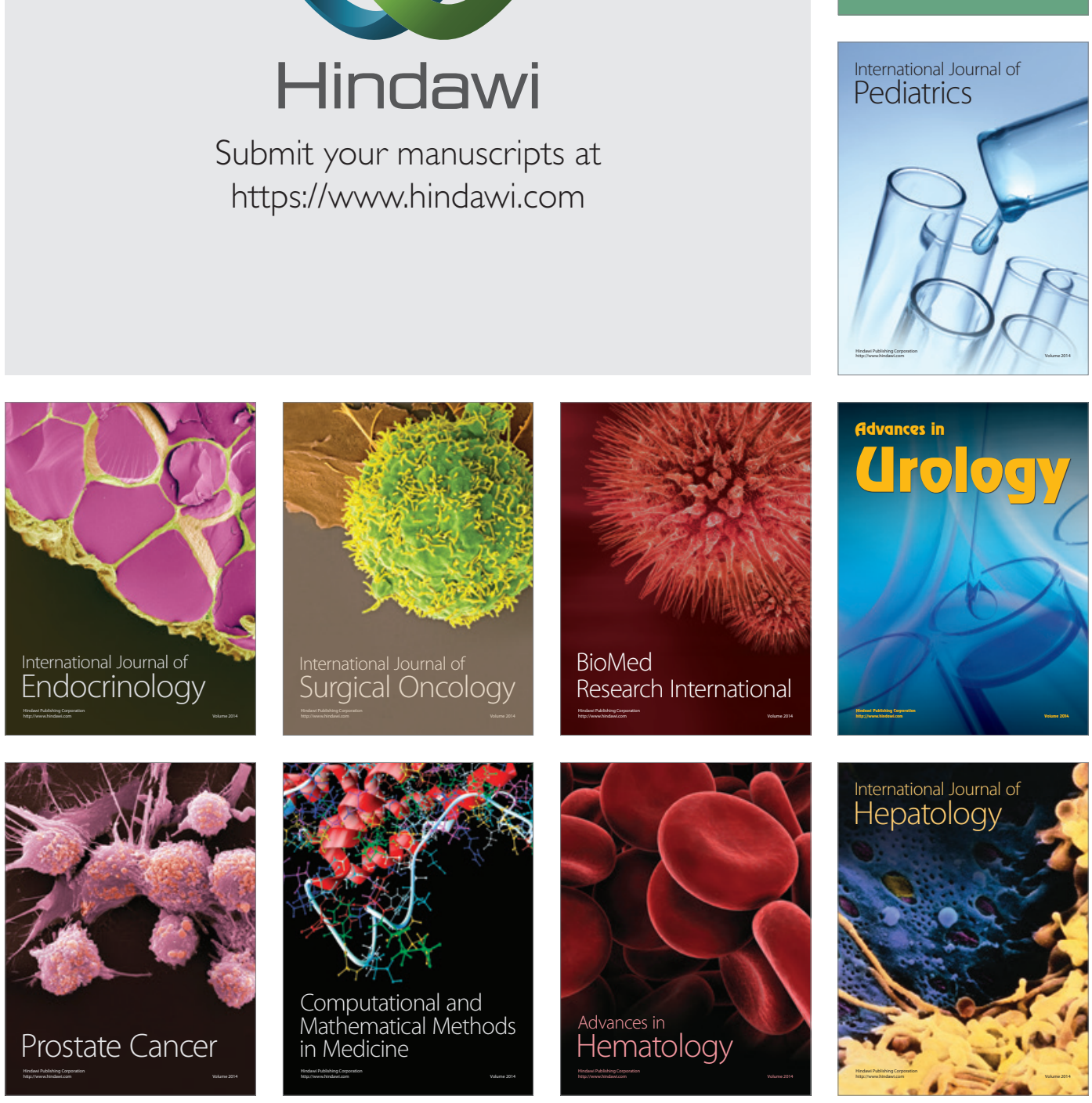